\title{
OSTEOARTHRITIS
}

\section{Control of human cartilage hypertrophic differentiation}

Disruption of articular cartilage homeostasis is important in osteoarthritis (OA) pathogenesis, key to which is activation of articular chondrocyte hypertrophic differentiation. Healthy articular cartilage is resistant to hypertrophic differentiation, whereas growth-plate cartilage is destined to differentiate in this way: could genetics explain these differences and possibly lead to a better understanding of the pathophysiology of OA? Research by Marcel Karperien and colleagues now identifies the bone morphogenetic protein antagonist Gremlin 1 (GREM1), as well as Frizzled-related protein (FRZB) and Dickkopf-related protein 1 (DKK1) -two Wnt antagonists-as natural brakes on hypertrophic differentiation in healthy cartilage.

Whole-genome expression microarray analysis of donor-paired healthy human articular and growth-plate cartilage revealed that expression levels of 35 genes differed more than 5 -fold between the tissues. Most differentially expressed were GREM1, FRZB and DKK1-all overexpressed in articular cartilage compared with growth-plate cartilage -as confirmed by immunohistochemistry.

Next, the investigators showed that recombinant GREM1, FRZB or DKK1

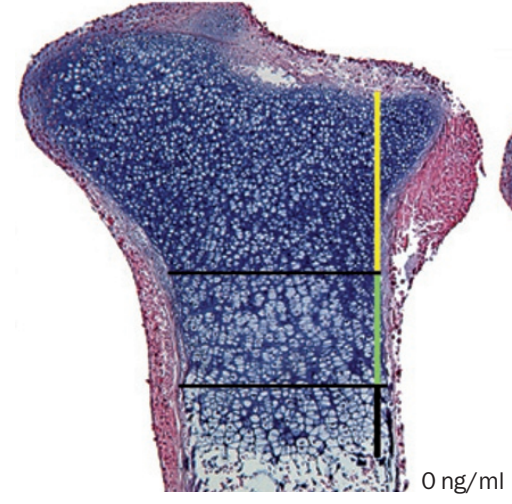

could inhibit terminal hypertrophic chondrocyte differentiation and mineralization of chondrogenicallydifferentiating human mesenchymal stem cells, without affecting chondrogenesis. Also, Grem1, Frzb and Dkk1 inhibited endochondral ossification in mouse explanted tibiae.

So, if GREM1, FRZB and DKK1 control hypertrophic chondrocyte differentiation in healthy articular cartilage are the genes encoding these proteins associated with OA risk? Associations between single nucleotide polymorphisms (SNPs) in or near GREM1 and DKK1 and radiographic hip or knee OA were investigated in two population-based cohorts. SNPrs12593365, located in a genomic control

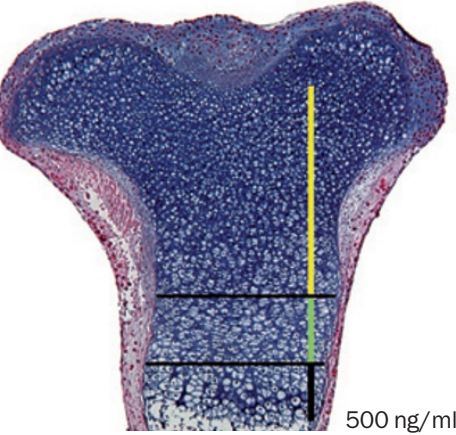

Mouse tibiae
(midsagittal
sections) cultured
for 7 days with
recombinant
Grem1, Frzb
and Dkk1 have
an increased
resting zone.
Image courtesy of
M. Karperien.

region of GREM1, was associated with a $20 \%$ reduced risk of radiographic hip OA.

"We succeeded in identifying specific markers to distinguish growth plate cartilage from articular cartilage and identified a mechanism likely to be involved in the regulation of articular cartilage homeostasis", explains Karperien. "This new area of research may lead to new diagnostic markers for $\mathrm{OA}$ and hopefully new routes for therapeutic intervention."

Jenny Buckland

Original article Leijten, J. C. et al. GREM1, FRZB and DKK1 are key regulators of human articular cartilage homeostasis. Arthritis Rheum. doi:10.1002/art.34535 